\title{
Almost periodic functionals and finite-dimensional representations
}

\author{
by \\ M. Filali (Oulu) and M. Sangani Monfared (Windsor, ON)
}

\begin{abstract}
We show that if $A$ is a $\mathrm{C}^{*}$-algebra and $\lambda \in A^{*}$ is a nonzero almost periodic functional which is a coordinate functional of a topologically irreducible involutive representation $\pi$, then $\operatorname{dim} \pi<\infty$. We introduce the RFD transform $\alpha_{A}: A \rightarrow U(A)$ of a Banach algebra $A$ and establish its universal property. We show that if $A$ has a bounded two-sided approximate identity, then almost periodic functionals on $A$ which are limits of coordinate functionals of finite-dimensional representations have lifts to almost periodic functionals on $U(A)$. Other connections with almost periodicity and harmonic analysis are also discussed.
\end{abstract}

1. Introduction and preliminaries. Let $A$ be a Banach algebra and $A^{*}$ be its dual space. Then $A^{*}$ carries a natural two-sided Banach $A$-module structure as follows: given $a \in A, f \in A^{*}$, the product $a \cdot f \in A^{*}$ is defined by the duality $\langle a \cdot f, b\rangle=\langle f, b a\rangle$ for all $b \in A$ (the product $f \cdot a \in A^{*}$ is defined similarly). A functional $\lambda \in A^{*}$ is called almost periodic if the map $A \rightarrow A^{*}$, $a \mapsto a \cdot \lambda$, is a compact linear operator. The space of all almost periodic functionals on $A$, denoted by $\operatorname{AP}(A)$, is a closed two-sided submodule of $A^{*}$. If $G$ is a locally compact group and $L^{1}(G)$ is the group algebra of $G$ (under the convolution product), then $\operatorname{AP}\left(L^{1}(G)\right)$ coincides with the $\mathrm{C}^{*}$-algebra of all continuous almost periodic functions on $G$, which we denote by $\operatorname{AP}(G)$. Among the extensive literature on almost periodic functionals, we can mention Dunkl and Ramirez [11, Young [26], Granirer [17, Lau and Wong [21, Duncan and Ülger [9], Dales and Lau [6].

We denote by $\mathscr{L}(E)$ the space of all continuous linear operators on a Banach space $E$. If $A$ is a Banach algebra, then by a continuous representation $\pi$ of $A$ on $E$, we mean a continuous algebra homomorphism $\pi: A \rightarrow \mathscr{L}(E)$. Given $x \in E, \phi \in E^{*}$, the coordinate functional $\pi_{x, \phi} \in A^{*}$

2010 Mathematics Subject Classification: 46H15, 43A60, 43A20, 22D10, 22D20.

Key words and phrases: almost periodic functionals, finite-dimensional representations, residually finite-dimensional algebras, compact groups, group algebra.

Received 26 June 2017; revised 22 September 2017.

Published online 7 May 2018. 
is defined by $\pi_{x, \phi}(a)=\langle\pi(a) x, \phi\rangle(a \in A)$. Perhaps the simplest connection between almost periodic functionals and representation theory is that every coordinate functional of a continuous finite-dimensional representation is almost periodic (Filali and Monfared [15, Lemma 2.3]).

In this paper we discuss several additional results related to almost periodic functionals and finite-dimensional representations. In Theorem 2.1 we show that if $A$ is an involutive Banach algebra, $\pi: A \rightarrow \mathscr{L}(H)$ is an involutive representation, and $\xi, \eta \in H$ are algebraically cyclic vectors such that $\pi_{\xi, \eta} \in \operatorname{AP}(A)$, then $\operatorname{dim} H<\infty$. In the remaining part of Section 2, several corollaries of this theorem are given. We show that if $A$ is a $\mathrm{C}^{*}$-algebra and $\lambda \in \operatorname{AP}(A)$ is a nonzero coordinate functional of a topologically irreducible involutive representation $\pi$, then $\operatorname{dim} \pi<\infty$ (Corollary 2.2). Furthermore, if $A$ is a unital $\mathrm{C}^{*}$-algebra, then every pure state $\lambda \in \mathrm{AP}(A)$ is a coordinate function of a finite-dimensional representation (Corollary 2.3). We will also show that if $G$ is a compact group, then every algebraically cyclic involutive representation of $L^{1}(G)$ is finite-dimensional (Corollary 2.4).

In Section 3 we introduce and study the properties of a canonical homomorphism $\alpha_{A}: A \rightarrow U(A)$, where $U(A)$ is a residually finite-dimensional (RFD) Banach algebra. We call $\alpha_{A}$ the RFD transform of $A$. We prove a universal property for the pair $\left(\alpha_{A}, U(A)\right)$, and show the existence of a bijection between finite-dimensional representations of $A$ and $U(A)$ (Theorem 3.4). The pair $\left(\alpha_{A}, U(A)\right)$ may thus be viewed as a Banach algebra analogue of the Bohr compactification of topological groups.

In analogy with the group case, one may ask whether almost periodic functionals on $A$ can be lifted to almost periodic functionals on the universal object $U(A)$. Theorem 4.4 shows that if $A$ has a bounded two-sided approximate identity, then this question has a positive answer for a large class of almost periodic functionals on $A$ (i.e., those which are limits of coordinate functionals of finite-dimensional representations). In Theorem 4.5 we show that $\alpha_{A}^{*}$ (the adjoint of $\alpha_{A}$ ) can be used to obtain almost periodic functionals on $A$. The connection between these ideas and harmonic analysis is briefly discussed in Theorem 4.6 and Example 4.7.

Now we review some notation and terminology. Throughout this paper, $G$ denotes a locally compact Hausdorff topological group equipped with a fixed left Haar measure, which we denote by $d s, d t$, etc. (When $G$ is compact, the Haar measure is normalized.) For the standard terminology on representation theory we refer to Dixmier [7] or Palmer [23]. We denote by $\widehat{G}$ the set of all (equivalence classes of) continuous, topologically irreducible, unitary representations of $G$ on Hilbert spaces. To simplify the notation we shall identify each equivalence class $[\pi]$ in $\widehat{G}$ with its representative $\pi$ (similar remark holds when we deal with representations of Banach algebras). 
If $\pi \in \widehat{G}$, then we can define an involutive topologically irreducible representation $\pi^{\prime}$ of $L^{1}(G)$ by $\pi^{\prime}(f)=\int_{G} f(s) \pi(s) d s, f \in L^{1}(G)$. We call $\pi^{\prime}$ the representation of $L^{1}(G)$ associated with $\pi$. Conversely, every nonzero topologically irreducible involutive representation of $L^{1}(G)$ is associated with some $\pi \in \widehat{G}$.

\section{2. $\operatorname{AP}(A)$ and involutive representations}

Theorem 2.1. Let $A$ be an involutive Banach algebra and $\pi: A \rightarrow \mathscr{L}(H)$ be an involutive representation of $A$ on a Hilbert space $H$. If $\xi, \eta \in H$ are algebraically cyclic vectors for $\pi$ such that $\pi_{\xi, \eta} \in \operatorname{AP}(A)$, then $\operatorname{dim} H<\infty$.

Proof. Let $\lambda=\pi_{\xi, \eta}$ and let $B_{A}$ denote the closed unit ball of $A$. Since $\lambda$ is almost periodic, the set $\left\{a \cdot \lambda: a \in B_{A}\right\}$ is relatively norm compact in $A^{*}$. The idea of the proof is to show that the set $W=\left\{\pi(a) \xi: a \in B_{A}\right\}$ is a relatively norm compact neighborhood of 0 in $H$. This will imply that $H$ is finite-dimensional (Dunford and Schwartz [10, Theorem IV.3.5]). It is easy to see that $W$ is a neighborhood of 0 in $H$. In fact, since $\xi$ is algebraically cyclic, we have $\pi(A) \xi=H$ and hence the map $\Gamma: A \rightarrow H, a \mapsto \pi(a) \xi$, is a continuous linear surjection. By the open mapping theorem, $\Gamma\left(B_{A}\right)=W$ contains an open neighborhood of 0 in $H$.

It remains to show that $W$ is relatively norm compact in $H$. This is equivalent to showing that for every sequence $\left\{a_{n}\right\}$ in $B_{A}$, the sequence $\left\{\pi\left(a_{n}\right) \xi\right\}$ in $W$ has a cluster point in $H$. Identifying $\left\{a_{n}\right\}$ with its natural image in $A^{* *}$, let $\Phi \in A^{* *}$ be a $w^{*}$-cluster point of $\left\{a_{n}\right\}$, and $\left\{a_{\alpha}\right\}$ be a subnet such that $a_{\alpha} \rightarrow \Phi$ in the $w^{*}$-topology of $A^{* *}$. Let $\widetilde{\pi}$ be the normal representation of $A^{* *}$ on $H$ extending $\pi$ (Filali, Neufang, and Monfared [14, Theorem 3.3]). We shall show that for a suitable subnet $\left\{a_{\alpha_{\beta}}\right\}$ of $\left\{a_{\alpha}\right\}$, we have

$$
\left\|\pi\left(a_{\alpha_{\beta}}\right) \xi-\widetilde{\pi}(\Phi) \xi\right\| \rightarrow 0 .
$$

Since $\lambda \in \operatorname{AP}(A)$, the set $\overline{B_{A} \cdot \lambda^{\|} \|_{A^{*}}}$ is compact, and the net $\left\{a_{\alpha} \cdot \lambda\right\}$ has a cluster point in $A^{*}$. Thus there exists some $f \in A^{*}$ and a subnet $\left\{a_{\alpha_{\beta}}\right\}$ of $\left\{a_{\alpha}\right\}$ such that $\left\|a_{\alpha_{\beta}} \cdot \lambda-f\right\|_{A^{*}} \rightarrow 0$. It is easy to check that $a_{\alpha_{\beta}} \cdot \lambda=$ $a_{\alpha_{\beta}} \cdot \pi_{\xi, \eta}=\pi_{\pi\left(a_{\alpha_{\beta}}\right) \xi, \eta}$, hence

$$
\begin{aligned}
\left\|a_{\alpha_{\beta}} \cdot \lambda-f\right\|_{A^{*}} & =\sup \left\{\left|\left\langle a_{\alpha_{\beta}} \cdot \lambda, b\right\rangle-\langle f, b\rangle\right|: b \in B_{A}\right\} \\
& =\sup \left\{\left|\left\langle\pi_{\pi\left(a_{\alpha_{\beta}}\right) \xi, \eta}, b\right\rangle-\langle f, b\rangle\right|: b \in B_{A}\right\} \\
& =\sup \left\{\left|\left(\pi(b)\left(\pi\left(a_{\alpha_{\beta}}\right) \xi\right) \mid \eta\right)-\langle f, b\rangle\right|: b \in B_{A}\right\} \rightarrow 0 .
\end{aligned}
$$

However, for given $b \in B_{A}$,

$$
\begin{aligned}
\left(\pi(b)\left(\pi\left(a_{\alpha_{\beta}}\right) \xi\right) \mid \eta\right) & =\left(\pi\left(a_{\alpha_{\beta}}\right) \xi \mid \pi(b)^{*} \eta\right) \\
& =\left(\widetilde{\pi}\left(a_{\alpha_{\beta}}\right) \xi \mid \pi\left(b^{*}\right) \eta\right) \rightarrow\left(\widetilde{\pi}(\Phi) \xi \mid \pi\left(b^{*}\right) \eta\right) .
\end{aligned}
$$


It follows from (2) and (3) that for every $b \in B_{A}$,

$$
\langle f, b\rangle=\left(\widetilde{\pi}(\Phi) \xi \mid \pi\left(b^{*}\right) \eta\right) .
$$

Thus using (2) and (4) we can write

$$
\sup \left\{\left|\left(\pi\left(a_{\alpha_{\beta}}\right) \xi \mid \pi\left(b^{*}\right) \eta\right)-\left(\widetilde{\pi}(\Phi) \xi \mid \pi\left(b^{*}\right) \eta\right)\right|: b \in B_{A}\right\} \rightarrow 0 .
$$

Since $B_{A}$ is stable under involution, we can rewrite (5) as

$$
\sup \left\{\left|\left(\pi\left(a_{\alpha_{\beta}}\right) \xi-\widetilde{\pi}(\Phi) \xi \mid \pi(b) \eta\right)\right|: b \in B_{A}\right\} \rightarrow 0 .
$$

Let $W^{\prime}=\left\{\pi(b) \eta: b \in B_{A}\right\}$. As we saw for $W$, since $\eta$ is algebraically cyclic, the open mapping theorem implies that $W^{\prime}$ contains an open neighborhood of 0 in $H$, say $B_{r}(0)$. It follows that

$$
\begin{aligned}
\left\|\pi\left(a_{\alpha_{\beta}}\right) \xi-\widetilde{\pi}(\Phi) \xi\right\| & =\sup _{\zeta \in H,\|\zeta\| \leq 1}\left|\left(\pi\left(a_{\alpha_{\beta}}\right) \xi-\widetilde{\pi}(\Phi) \xi \mid \zeta\right)\right| \\
& =\frac{2}{r} \sup _{\zeta \in H,\|\zeta\| \leq 1}\left|\left(\pi\left(a_{\alpha_{\beta}}\right) \xi-\widetilde{\pi}(\Phi) \xi \mid r \zeta / 2\right)\right| \\
& =\frac{2}{r} \sup _{\zeta^{\prime} \in H,\left\|\zeta^{\prime}\right\| \leq r / 2}\left|\left(\pi\left(a_{\alpha_{\beta}}\right) \xi-\widetilde{\pi}(\Phi) \xi \mid \zeta^{\prime}\right)\right| \\
& \leq \frac{2}{r} \sup _{\zeta \in H,\|\zeta\|<r}\left|\left(\pi\left(a_{\alpha_{\beta}}\right) \xi-\widetilde{\pi}(\Phi) \xi \mid \zeta\right)\right| \\
\text { (since } \left.B_{r}(0) \subset W^{\prime}\right) & \leq \frac{2}{r} \sup _{\zeta \in W^{\prime}}\left|\left(\pi\left(a_{\alpha_{\beta}}\right) \xi-\widetilde{\pi}(\Phi) \xi \mid \zeta\right)\right| \\
(\text { by } 60) & =\frac{2}{r} \sup \left\{\left|\left(\pi\left(a_{\alpha_{\beta}}\right) \xi-\widetilde{\pi}(\Phi) \xi \mid \pi(b) \eta\right)\right|: b \in B_{A}\right\} \rightarrow 0 .
\end{aligned}
$$

This proves (1), and hence completes the proof.

Corollary 2.2. Let $A$ be a $\mathrm{C}^{*}$-algebra and $\lambda \in \mathrm{AP}(A)$ be nonzero. If $\lambda$ is a coordinate functional of a topologically irreducible involutive representation $\pi: A \rightarrow \mathscr{L}(H)$, then $\operatorname{dim} H<\infty$.

Proof. Let $\lambda=\pi_{\xi, \eta}$, with $\xi, \eta \in H$. By Kadison's irreducibility theorem (see Dixmier [7, Corollaire 2.8.4]), $\pi$ is algebraically irreducible, and hence, both $\xi, \eta$ are algebraically cyclic vectors for $\pi$. By Theorem 2.1. $\operatorname{dim} H<\infty$.

Let $A$ be unital $\mathrm{C}^{*}$-algebra. The set $\mathscr{S}$ of all states on $A$ is a $w^{*}$-compact convex subset of the unit ball of $A^{*}$. The extreme points of $\mathscr{S}$ (which exist by the Krein-Milman theorem) are called the pure states on $A$.

COROllary 2.3. If $A$ is a unital $\mathrm{C}^{*}$-algebra, then every pure state $\lambda \in \operatorname{AP}(A)$ is a coordinate functional of a finite-dimensional involutive representation of $A$. 
Proof. Since $\lambda$ is positive, by the GNS construction there exists an involutive representation $\pi: A \rightarrow \mathscr{L}(H)$ such that $\lambda=\pi_{\xi, \xi}$ for some $\xi \in H$. Since $\lambda$ is a pure state, $\pi$ is topologically irreducible (Dixmier [7, Proposition 2.5.4]). The result follows from Corollary 2.2.

We recall that if $G$ is a compact group, then every topologically irreducible involutive representation of $L^{1}(G)$ is finite-dimensional. As our final corollary of Theorem 2.1, we state the following.

COROLlaRY 2.4. If $G$ is a compact group, then every algebraically cyclic involutive representation of $L^{1}(G)$ is finite-dimensional.

Proof. Let $\pi: L^{1}(G) \rightarrow \mathscr{L}(H)$ be an involutive representation with an algebraically cyclic vector $\xi \in H$. Then $\pi_{\xi, \xi} \in C(G)=\operatorname{AP}(G)=\operatorname{AP}\left(L^{1}(G)\right)$. The result follows from Theorem 2.1.

3. The RFD transform. Let $G$ be a locally compact group. It is well-known that the Bohr (or almost periodic) compactification of $G$, denoted by $b(G)$, can be obtained using finite-dimensional unitary representations of $G$ (see for example, Dixmier [7, Théorème 16.1.1]). The compact group $b(G)$ has several properties of interest, among which we can mention the following: (i) $b(G)$ has sufficiently many finite-dimensional unitary representations, (ii) there exists a canonical continuous homomorphism $\alpha: G \rightarrow b(G)$ such that given a compact group $H$ and a continuous homomorphism $\alpha^{\prime}: G \rightarrow H$, one can find a unique continuous homomorphism $\beta: b(G) \rightarrow H$ with $\alpha^{\prime}=\beta \circ \alpha$ (universal property), (iii) there exists a bijection between the (equivalence classes of) finite-dimensional unitary representations of $G$ and $b(G)$, (iv) a bounded continuous function $f$ on $G$ is almost periodic if and only if there exists a continuous function $g$ on $b(G)$ such that $f=g \circ \alpha$.

The Bohr compactification is a powerful tool in harmonic analysis and its applications. In this section we discuss an analogous construction in the category of Banach algebras. The relevance of this construction to the study of almost periodic functionals will be discussed in the next section. For our purposes, the property that will play the role of 'compactness' for Banach algebras is the existence of sufficiently many finite-dimensional representations. This property has been studied extensively for $\mathrm{C}^{*}$-algebras (though perhaps not with a view toward almost periodic functionals), and $\mathrm{C}^{*}$-algebras having this property are called residually finite-dimensional (RFD). From the relevant literature we can mention Choi [3], Goodearl and Menal [16], Exel and Loring [12], Pestov [24, Archbold [1], Dadarlat [4, and Lin [22]. Since in this paper we are interested in both involutive and noninvolutive algebras, we shall make precise our meaning of an RFD Banach algebra (here and below, the phrases pertaining to the involutive case appear in brackets [ ]). 
Definition 3.1. An [involutive] Banach algebra $A$ is residually finitedimensional (RFD) if for every $a \in A$ we have $\|a\|=\sup _{\pi}\|\pi(a)\|$, where the supremum is taken over all [irreducible involutive] finite-dimensional representations $\pi$ of $A$ with $\|\pi\| \leq 1$.

If $X$ is a locally compact space, then every closed subalgebra $A$ of $C_{0}(X)$ is RFD, since for each $f \in A,\|f\|=\sup _{x \in X}|f(x)|$. The $\mathrm{C}^{*}$-algebra $C_{0}\left(X, \mathbb{M}_{n}(\mathbb{C})\right)$ is also RFD (cf. Blackadar [2, IV.1.4.1]). Choi [3, Theorem 7] has shown that the full $\mathrm{C}^{*}$-algebra $\mathrm{C}^{*}(F)$ of any free group $F$ with a countable set of generators is RFD. In general, the RFD property is preserved under the formation of direct sums and passage to closed subalgebras. The group algebra $L^{1}(G)$ and the Fourier algebra $A(G)$ (Eymard [13]) are, in general, not RFD (see Remark 3.3).

Let us note that in the definition of an involutive RFD Banach algebra, the condition of irreducibility of involutive representations is not an additional restriction, since finite-dimensional involutive representations are direct sums of irreducible ones (Dixmier [7, (2.3.5)]). It follows that, if $A$ is a $\mathrm{C}^{*}$-algebra, then $A$ is RFD if and only if $A$ has a separating family of finite-dimensional involutive representations (the equivalence follows easily from Takesaki [25, Proposition I.5.3]). This shows that for $\mathrm{C}^{*}$-algebras, Definition 3.1 agrees with the one usually found in the literature on $\mathrm{C}^{*}$-algebras.

To every Banach algebra we can associate an RFD Banach algebra as follows. Let $A$ be an [involutive] Banach algebra and let $\left\{\left(\pi_{i}, H_{i}\right)\right\}_{i \in I}$ be the family of all [irreducible involutive] finite-dimensional representations of $A$ with $\left\|\pi_{i}\right\| \leq 1$. We call the continuous homomorphism

$$
\alpha_{A}: A \rightarrow \ell^{\infty}-\bigoplus_{i \in I} \mathscr{L}\left(H_{i}\right), \quad \alpha_{A}(a)=\left(\pi_{i}(a)\right)_{i \in I},
$$

the RFD transform of $A$ (the Banach algebra direct sum $\ell^{\infty}-\bigoplus_{i \in I} \mathscr{L}\left(H_{i}\right)$ has coordinatewise operations and is equipped with the supremum norm). We denote the closure of $\alpha_{A}(A)$ in $\ell^{\infty}-\bigoplus_{i \in I} \mathscr{L}\left(H_{i}\right)$ by $U(A)$, and call it the $R F D$ Banach algebra associated with $A$ (see Theorem 3.4). If $A$ does not have any nonzero finite-dimensional representations, then $U(A)=\{0\}$.

EXAMPLE 3.2. Let $G$ be either a compact group or a locally compact abelian group, and let $L^{1}(G)$ be the group algebra of $G$ under the convolution product. Then the set of nonzero finite-dimensional irreducible involutive representations of $L^{1}(G)$ can be written as $\left\{\left(\bar{\pi}^{\prime}, H_{\pi}\right)\right\}_{\pi \in \widehat{G}}$ (where $\bar{\pi}$ is the representation of $G$ conjugate to $\pi$, and $\bar{\pi}^{\prime}$ is the representation of $L^{1}(G)$ associated with $\bar{\pi})$. Then for $f \in L^{1}(G)$ and $\pi \in \widehat{G}$, we have

$$
\alpha_{L^{1}}(f)(\pi)=\bar{\pi}^{\prime}(f)=\int_{G} f(s) \bar{\pi}(s) d s=\widehat{f}(\pi),
$$

where $\widehat{f}$ is the Fourier transform of $f[18,(28.34)]$. Thus $\left.\alpha_{L^{1}}(f)\right|_{\widehat{G}}=\widehat{f}$ and 
$\left\|\alpha_{L^{1}}(f)\right\|_{\infty}=\|\widehat{f}\|_{\infty}$ for all $f \in L^{1}(G)$. At the end of this section, $U\left(L^{1}(G)\right)$ will be characterized when $G$ is either abelian or compact.

REMARK 3.3. If $A$ is RFD, then the homomorphism $\alpha_{A}$ is an isometric isomorphism onto its image. In particular, an RFD Banach algebra is Arens regular (Duncan and Hosseiniun [8], Dales [5]), and an involutive RFD Banach algebra is a $\mathrm{C}^{*}$-algebra.

We now state the main result of this section.

Theorem 3.4. Let $A$ be an [involutive] Banach algebra. Then $U(A)$ is an [involutive] RFD Banach algebra with the following properties:

(i) If $B$ is an [involutive] RFD Banach algebra and $\alpha^{\prime}: A \rightarrow B$ is an [involutive] homomorphism with $\left\|\alpha^{\prime}\right\| \leq 1$, then there exists a unique [involutive] homomorphism $\beta: U(A) \rightarrow B$ such that $\|\beta\| \leq 1$ and $\alpha^{\prime}=$ $\beta \circ \alpha_{A}$ (universal property).

(ii) Let $\mathscr{C}$ and $\check{\mathscr{C}}$ be the sets of all [irreducible involutive] finite-dimensional representations of $A$ and $U(A)$ respectively, with norms $\leq 1$. Then the map

$$
\check{\mathscr{C}} \rightarrow \mathscr{C}, \quad \check{\pi} \mapsto \pi:=\check{\pi} \circ \alpha_{A},
$$

is a bijection between $\check{\mathscr{C}}$ and $\mathscr{C}$. Moreover, $\pi$ is irreducible if and only if $\check{\pi}$ is irreducible.

Up to isometric isomorphism, the pair $\left(\alpha_{A}, U(A)\right)$ is uniquely determined by the universal property (i).

Proof. We will give the proof for involutive algebras (the proof for the noninvolutive case is similar). Let $\left\{\left(\pi_{i}, H_{i}\right)\right\}_{i \in I}$ denote the family of all irreducible involutive finite-dimensional representations of $A$. For each $i \in I$, let $\operatorname{pr}_{i}: U(A) \rightarrow \mathscr{L}\left(H_{i}\right)$ be the projection onto the $i$ th coordinate. Then $\mathrm{pr}_{i}$ is an involutive finite-dimensional representation of $U(A)$. Since $\pi_{i}$ is irreducible and $\operatorname{pr}_{i}\left(\alpha_{A}(a)\right)=\pi_{i}(a)$ for all $a \in A$, it follows that $\operatorname{pr}_{i}$ is also irreducible. Moreover, by the definition of $U(A)$, if $T=\left(T_{i}\right)_{i \in I} \in U(A)$, then $\|T\|=\sup _{i \in I}\left\|\operatorname{pr}_{i}(T)\right\|$, and thus $U(A)$ is RFD.

(i) Let $\left\{\left(\theta_{j}, H_{j}^{\prime}\right)\right\}_{j \in J}$ be the family of all irreducible involutive finitedimensional representations of $B$. For each $j \in J$, the map

$$
\rho_{j}:=\theta_{j} \circ \alpha^{\prime}
$$

is an involutive finite-dimensional representation of $A$ on $H_{j}^{\prime}$. Let

$$
\rho_{j}=\bigoplus_{k=1}^{N_{j}} \bigoplus^{n_{j_{k}}} \rho_{j_{k}}
$$

be a decomposition of $\rho_{j}$ into involutive irreducible representations of $A$ (where $\bigoplus^{n_{j_{k}}} \rho_{j_{k}}=\rho_{j_{k}} \oplus \cdots \oplus \rho_{j_{k}}, n_{j_{k}}$ summands). Each $\rho_{j_{k}}$ must then be 
unitarily equivalent to one of the representations in $\left\{\left(\pi_{i}, H_{i}\right)\right\}_{i \in I}$, say $\pi_{i_{j_{k}}}$. Hence

$$
\rho_{j} \cong \bigoplus_{k=1}^{N_{j}} \bigoplus^{n_{j_{k}}} \pi_{i_{j_{k}}}
$$

Let $I_{j}=\left\{i_{j_{k}}: k=1, \ldots, N_{j}\right\}$, and $\operatorname{pr}_{I_{j}}: U(A) \rightarrow \bigoplus_{k=1}^{N_{j}} \mathscr{L}\left(H_{i_{j_{k}}}\right)$ be the projection of $U(A)$ onto its $I_{j}$ th component. Let $\sigma_{j}$ be the 'amplification' map defined by

$$
\sigma_{j}: \bigoplus_{k=1}^{N_{j}} \mathscr{L}\left(H_{i_{j_{k}}}\right) \rightarrow \bigoplus_{k=1}^{N_{j}} \bigoplus^{n_{j_{k}}} \mathscr{L}\left(H_{i_{j_{k}}}\right), \quad \bigoplus_{k=1}^{N_{j}} T_{k} \mapsto \bigoplus_{k=1}^{N_{j}} \bigoplus^{n_{j_{k}}} T_{k} .
$$

Next, let

$$
\gamma_{j}: \bigoplus_{k=1}^{N_{j}} \bigoplus^{n_{j_{k}}} \mathscr{L}\left(H_{i_{j_{k}}}\right) \rightarrow \mathscr{L}\left(H_{j}^{\prime}\right)
$$

be the isometric involutive homomorphism such that

$$
\rho_{j}=\gamma_{j} \circ \bigoplus_{k=1}^{N_{j}} \bigoplus^{n_{j_{k}}} \pi_{i_{j_{k}}}
$$

and define

$$
\beta_{j}^{\prime}=\gamma_{j} \circ \sigma_{j} \circ \operatorname{pr}_{I_{j}} .
$$

Then $\beta_{j}^{\prime}: U(A) \rightarrow \mathscr{L}\left(H_{j}^{\prime}\right)$ is an involutive homomorphism, and for every $a \in A$,

$$
\left(\beta_{j}^{\prime} \circ \alpha_{A}\right)(a)=\gamma_{j} \circ \sigma_{j}\left(\operatorname{pr}_{I_{j}}\left(\alpha_{A}(a)\right)\right)=\gamma_{j}\left(\bigoplus_{k=1}^{N_{j}} \bigoplus^{n_{j_{k}}} \pi_{i_{j_{k}}}(a)\right)=\rho_{j}(a) .
$$

Consider the involutive homomorphism

$$
\beta^{\prime}=\left(\beta_{j}^{\prime}\right)_{j \in J}: U(A) \rightarrow \ell^{\infty}-\bigoplus_{j \in J} \mathscr{L}\left(H_{j}^{\prime}\right), \quad T \mapsto\left(\beta_{j}^{\prime}(T)\right)_{j \in J} .
$$

Since $B$ is RFD, it follows from the definition that $\alpha_{B}$ is an involutive isometric isomorphism of $B$ onto $\alpha_{B}(B)$, and thus $B \cong \alpha_{B}(B)=U(B)$. Therefore by (9), (10), and the density of $\alpha_{A}(A)$ in $U(A)$, we can write $\beta^{\prime}(U(A)) \subset \alpha_{B}(B)$. We claim that the map $\beta$ defined by

$$
\beta:=\alpha_{B}^{-1} \circ \beta^{\prime}: U(A) \rightarrow B
$$

is the required involutive homomorphism satisfying $\alpha^{\prime}=\beta \circ \alpha_{A}$. In fact, for every $a \in A$, using $(9)$ and $(10)$ we can write

$$
\beta^{\prime}\left(\alpha_{A}(a)\right)=\left(\beta_{j}^{\prime}\left(\alpha_{A}(a)\right)\right)_{j \in J}=\left(\rho_{j}(a)\right)_{j \in J}=\left(\theta_{j}\left(\alpha^{\prime}(a)\right)\right)_{j \in J}=\alpha_{B}\left(\alpha^{\prime}(a)\right) .
$$

In other words, $\left(\alpha_{B}^{-1} \circ \beta^{\prime}\right)\left(\alpha_{A}(a)\right)=\alpha^{\prime}(a)$ for all $a \in A$, thus by (11), $\beta \circ \alpha_{A}=\alpha^{\prime}$. The uniqueness of $\beta$ is immediate because $\alpha_{A}(A)$ is dense in $U(A)$. 
(ii) It is easy to verify that the map $\check{\pi} \mapsto \pi$ preserves the equivalence classes and is well-defined. To prove surjectivity, let $(\pi, H) \in \mathscr{C}$. Keeping the notation as in the proof of (i), we must have $(\pi, H)=\left(\pi_{i}, H_{i}\right)$ for some $i \in I$. Since the $i$ th projection $\operatorname{pr}_{i}: U(A) \rightarrow \mathscr{L}\left(H_{i}\right)$ belongs to $\check{\mathscr{C}}$, by letting $\check{\pi}=\operatorname{pr}_{i}$ we obtain $\check{\pi} \circ \alpha_{A}=\pi$. To prove injectivity, suppose $\check{\pi}_{1}, \check{\pi}_{2} \in \check{\mathscr{C}}$ and $\check{\pi}_{1} \circ \alpha_{A} \cong$ $\check{\pi}_{2} \circ \alpha_{A}$. Then for a unitary operator $V, \check{\pi}_{2}\left(\alpha_{A}(a)\right)=V^{-1} \check{\pi}_{1}\left(\alpha_{A}(a)\right) V$ for all $a \in A$. Since $\alpha_{A}(A)$ is norm dense in $U(A)$, it follows that $\check{\pi}_{1} \cong \check{\pi}_{2}$. The fact that irreducibility of $\pi$ and of $\check{\pi}$ are equivalent follows immediately from the density of $\alpha_{A}(A)$ in $U(A)$.

The uniqueness of $\left(\alpha_{A}, U(A)\right)$ (up to isometric isomorphism) is an easy consequence of its universal property (i); the details are left to the reader.

We recall that an involutive Banach algebra $A$ is called symmetric if the Gelfand transform of $A$ is an involutive homomorphism. Examples include group algebras and $\mathrm{C}^{*}$-algebras. The following result (whose proof is left to the reader) gives an alternative description of $\left(\alpha_{A}, U(A)\right)$ when $A$ is a commutative symmetric Banach algebra.

TheOREM 3.5. Let $A$ be a commutative symmetric Banach algebra with nonempty spectrum, and let $\mathscr{G}: A \rightarrow C_{0}(\sigma(A))$ be the Gelfand transform. Then there exists an isometric involutive isomorphism $\Psi: \overline{\mathscr{G}(A)} \rightarrow U(A)$, such that $\alpha_{A}=\Psi \circ \mathscr{G}$.

Corollary 3.6.

(i) If $A$ is a commutative $C^{*}$-algebra, then $U(A) \cong C_{0}(\sigma(A)) \cong A$, with $\alpha_{A}$ being the Gelfand transform.

(ii) If $G$ is a locally compact abelian group, then $U\left(L^{1}(G)\right) \cong C_{0}(\widehat{G})$, with $\alpha_{L^{1}}$ being the Fourier transform.

Let $G$ be an arbitrary compact group and let $c_{0}-\bigoplus_{\pi \in \widehat{G}} \mathscr{L}\left(H_{\pi}\right)$ be the space of all $\left(T_{\pi}\right)_{\pi \in \widehat{G}} \in \ell^{\infty}-\bigoplus_{\pi \in \widehat{G}} \mathscr{L}\left(H_{\pi}\right)$ such that $\left\{\pi \in \widehat{G}:\left\|T_{\pi}\right\| \geq \epsilon\right\}$ is finite for all $\epsilon>0$.

THEOREM 3.7. If $G$ is a compact group, then

$$
U\left(L^{1}(G)\right) \cong c_{0}-\bigoplus_{\pi \in \widehat{G}} \mathscr{L}\left(H_{\pi}\right) .
$$

Proof. Consider the isometric linear function $\Phi\left(\alpha_{L^{1}}(f)\right)=\widehat{f}$, mapping $\alpha_{L^{1}}\left(L^{1}(G)\right)$ to $c_{0}-\bigoplus_{\pi \in \widehat{G}} \mathscr{L}\left(H_{\pi}\right)$ (see Example 3.2 . Since $\alpha_{L^{1}}\left(L^{1}(G)\right)$ is dense in $U\left(L^{1}(G)\right)$, this map has a unique extension to an isometric linear function $\bar{\Phi}$ from $U\left(L^{1}(G)\right)$ to $c_{0}-\bigoplus_{\pi \in \widehat{G}} \mathscr{L}\left(H_{\pi}\right)$. Furthermore, since $\widehat{L^{1}(G)}$ is norm dense in $c_{0}-\bigoplus_{\pi \in \widehat{G}} \mathscr{L}\left(H_{\pi}\right)$ [18, Theorem 28.40] and the image of $\bar{\Phi}$ must be complete, it follows that $\bar{\Phi}$ maps $U\left(L^{1}(G)\right)$ isometrically onto $c_{0}-\bigoplus_{\pi \in \widehat{G}} \mathscr{L}\left(H_{\pi}\right)$, as we wanted to show. 
4. RFD transform and almost periodicity. In this section we discuss how the RFD transform $\alpha_{A}$ can be used in the study of almost periodic functionals. We start with a simple but interesting result which shows that almost periodic functionals on an RFD Banach algebra determine the norm of the algebra.

Theorem 4.1. If $A$ is an RFD Banach algebra, then for all $a \in A$,

$$
\|a\|=\sup \{|\lambda(a)|: \lambda \in \operatorname{AP}(A),\|\lambda\| \leq 1\} .
$$

Proof. Given $a \in A$ and $\epsilon>0$, there exists a finite-dimensional representation $(\pi, H)$ of $A$ with $\|\pi\| \leq 1$ such that $\|\pi(a)\|>\|a\|-\epsilon$. Thus we can find $\xi, \eta \in H$ with $\|\xi\|,\|\eta\| \leq 1$ such that $\left|\pi_{\xi, \eta}(a)\right|=|(\pi(a) \xi \mid \eta)| \geq\|a\|-\epsilon$. Since $\pi_{\xi, \eta} \in \operatorname{AP}(A)$ (Filali-Monfared [15, Theorem 2.3]) and $\left\|\pi_{\xi, \eta}\right\| \leq 1$, the result follows.

We recall from Section 3 that if $\alpha: G \rightarrow b(G)$ is the Bohr compactification of a topological group $G$, then a bounded continuous function $f$ on $G$ is almost periodic exactly when $f$ can be 'lifted' to a continuous function $g$ on $b(G)$ (i.e., $f=g \circ \alpha$ ). Note that such a function $g$ is automatically almost periodic since $b(G)$ is compact. This raises a natural question: if $\alpha_{A}: A \rightarrow U(A)$ is the RFD transform of a Banach algebra $A$, is a functional $f \in A^{*}$ almost periodic if and only if there exists an almost periodic functional $g \in U(A)^{*}$ such that $f=g \circ \alpha_{A}$ ?

The 'if' part of this question has a positive answer which follows from the following result of Duncan and Ülger:

Lemma 4.2. If $A$ and $B$ are Banach algebras and $\phi: A \rightarrow B$ is a continuous homomorphism, then $\phi^{*}(\mathrm{AP}(B)) \subset \mathrm{AP}(A)$, where $\phi^{*}$ is the adjoint of $\phi$.

The lemma follows from [9, proof of Proposition 4.2, first part] (where $\phi$ need not be surjective). Thus if $f \in A^{*}$ and $g \in \operatorname{AP}(U(A))$ are such that $g \circ \alpha_{A}=f$, then $f=\alpha_{A}^{*}(g) \in \operatorname{AP}(A)$.

REMARK 4.3. Unlike the group case, where every continuous function on $b(G)$ is almost periodic, the set of almost periodic functionals on $U(A)$ is in general a proper subspace of $U(A)^{*}$. As an example, let $X$ be an infinite compact space and $C(X)$ be the space of all continuous functions on $X$. By Corollary 3.6. $U(C(X)) \cong C(X)$, and the RFD transform of $C(X)$ can be identified with the identity map. Duncan and Ülger 9, Proposition 3.5] have shown that $\operatorname{AP}(C(X))=\ell_{1}(X)$, which is a proper subspace of $C(X)^{*}=M(X)$.

In the following we will show that the 'only if' part of the above question has a positive answer at least in the case of AP-functionals which are limits of coordinate functionals of continuous finite-dimensional representations. 
We denote by $\mathfrak{F}\left(A^{*}\right)$ the linear subspace of $A^{*}$ consisting of all coordinate functionals of continuous finite-dimensional representations of $A$, and $\overline{\mathfrak{F}\left(A^{*}\right)}$ its closure in the norm topology of $A^{*}$.

TheOREM 4.4. Let $A$ be a Banach algebra with a bounded two-sided approximate identity. If $f \in \overline{\mathfrak{F}\left(A^{*}\right)}$, then there exists a unique almost periodic functional $g$ on $U(A)$ such that $f=g \circ \alpha_{A}$.

Proof. Let $\left\{f_{n}\right\}_{n}$ be a sequence in $\mathfrak{F}\left(A^{*}\right)$ such that $\left\|f_{n}-f\right\|_{A^{*}} \rightarrow 0$. Let $X_{n}$ be the linear subspace of $A^{*}$ generated by the set $A \cdot f_{1} \cup \cdots \cup A \cdot f_{n}$. Since each $A \cdot f_{j}$ is finite-dimensional, so is $X_{n}$. Let $X=\bar{\bigcup}_{n=1}^{\infty} X_{n}$, where the closure is in the norm topology of $A^{*}$, and let $\left\{e_{\alpha}\right\}$ be a bounded twosided approximate identity of $A$. Then for each $n, f_{n} \in X_{n}$. In fact, on the one hand we have $\lim _{\alpha} e_{\alpha} \cdot f_{n}=f_{n}$ in the $w^{*}$-topology of $A^{*}$, and on the other hand, since $f_{n} \in \operatorname{AP}(A)$, there exist $g \in A^{*}$ and a subnet $\left\{e_{\alpha_{\beta}} \cdot f_{n}\right\}$ such that $\lim _{\beta} e_{\alpha_{\beta}} \cdot f_{n}=g$ in the norm topology of $A^{*}$. Thus $f_{n}=g$, and because $X_{n}$ is closed in the norm topology of $A^{*}$, we obtain $f_{n} \in X_{n}$. It follows that $f \in X$.

Let $L: A \rightarrow \mathscr{L}\left(A^{*}\right)$ be the continuous representation of $A$ on $A^{*}$ defined by $L(a) h=a \cdot h$, where $a \in A, h \in A^{*}$. Since each $X_{n}$ is an invariant subspace of $L$, so is $X$, and hence we can define the Banach algebra $B=\overline{\left\{\left.L(a)\right|_{X}: a \in A\right\}} \subset \mathscr{L}(X)$, where the closure is in the norm topology of $\mathscr{L}(X)$. Since each operator $T \in B$ is a norm limit of a sequence $\left\{\left.L\left(a_{k}\right)\right|_{X}\right\}_{k \in \mathbb{N}}$ of operators, it follows that for $x \in X_{n}, T x=\lim _{k} L\left(a_{k}\right) x$ $\in X_{n}$. Thus for each $n \geq 1$, the mapping $\pi_{n}: B \rightarrow \mathscr{L}\left(X_{n}\right),\left.T \mapsto T\right|_{X_{n}}$, is a finite-dimensional representation of $B$ with $\left\|\pi_{n}\right\| \leq 1$. We claim that $B$ is RFD. To prove this it suffices to show that $\|T\| \leq \sup _{n}\left\|\pi_{n}(T)\right\|$ for every $T \in B$. Since $\bigcup_{n=1}^{\infty} X_{n}$ is dense in $X$, we have $\|T\|=\left\|\left.T\right|_{\bigcup_{n=1}^{\infty} X_{n}}\right\|$. If $x \in \bigcup_{n=1}^{\infty} X_{n}$, then $x \in X_{n}$ for some $n$, and therefore

$$
\left\|\left.T\right|_{\cup_{n=1}^{\infty} X_{n}}(x)\right\|=\left\|\left.T\right|_{X_{n}} x\right\|=\left\|\pi_{n}(T) x\right\| \leq\left\|\pi_{n}(T)\right\|\|x\| \leq \sup _{n}\left\|\pi_{n}(T)\right\|\|x\| .
$$

It follows that $\|T\|=\left\|\left.T\right|_{\bigcup_{n=1}^{\infty} X_{n}}\right\| \leq \sup _{n}\left\|\pi_{n}(T)\right\|$; thus $B$ is RFD. Since the mapping $L^{\prime}: A \rightarrow B, L^{\prime}(a)=\left.L(a)\right|_{X}$ is a homomorphism with $\left\|L^{\prime}\right\| \leq 1$, it follows from the universal property of the pair $\left(\alpha_{A}, U(A)\right)$ (Theorem 3.4) that there exists a homomorphism $\beta: U(A) \rightarrow B,\|\beta\| \leq 1$, such that $\beta \circ \alpha_{A}=L^{\prime}$.

Now let $\Psi \in A^{* *}$ be a $w^{*}$-cluster point of $\left\{e_{\alpha}\right\}$ in $A^{* *}$ and define $f \otimes \Psi \in B^{*}$ by $\langle f \otimes \Psi, T\rangle=\langle\Psi, T f\rangle(T \in B)$. Define $g=\beta^{*}(f \otimes \Psi) \in U(A)^{*}$. Then $g$ is a lift of $f$, since for all $a \in A$,

$$
\begin{aligned}
g \circ \alpha_{A}(a) & =\left\langle\beta^{*}(f \otimes \Psi), \alpha_{A}(a)\right\rangle=\left\langle f \otimes \Psi, L^{\prime}(a)\right\rangle \\
& =\langle\Psi, a \cdot f\rangle=\langle\Psi \cdot a, f\rangle=\langle a, f\rangle,
\end{aligned}
$$


where to obtain the last identity we used the fact that $\Psi$ is a mixed identity in $A^{* *}$. To complete the proof it remains to show that $g$ is almost periodic. But since $\beta$ is a continuous homomorphism, it suffices to show that $f \otimes \Psi$ is almost periodic on $B$ (Lemma 4.2). However, since

$$
\left\|f_{n} \otimes \Psi-f \otimes \Psi\right\|_{B^{*}}=\left\|\left(f_{n}-f\right) \otimes \Psi\right\|_{B^{*}} \leq\left\|f_{n}-f\right\|_{A^{*}}\|\Psi\| \rightarrow 0,
$$

it suffices to show that each $f_{n} \otimes \Psi$ is almost periodic. If $\Psi_{n}=\left.\Psi\right|_{X_{n}}$, then it is easy to check that $f_{n} \otimes \Psi=\left(\pi_{n}\right)_{f_{n}, \Psi_{n}}$, that is, $f_{n} \otimes \Psi$ is a coordinate functional of $\pi_{n}$ and hence $f_{n} \otimes \Psi \in \operatorname{AP}(B)$. The uniqueness of $g$ follows from the density of $\alpha_{A}(A)$ in $U(A)$.

In the remainder of this section we discuss our final application of the RFD transform $\alpha_{A}: A \rightarrow U(A)$ to the study of almost periodic functionals. We begin by explaining the required terminology. Let $A$ be a Banach algebra and $\left\{\left(\pi_{i}, H_{i}\right)\right\}_{i \in I}$ be the family of all finite-dimensional representations of $A$ with $\left\|\pi_{i}\right\| \leq 1$ (as before, if $A$ is involutive, the representations are assumed to be irreducible and involutive). If $T$ is a square matrix, then we let $|T|=$ $\left(T T^{*}\right)^{1 / 2}$ and $\|T\|_{1}=\operatorname{tr}(|T|)$. For each $i \in I$, letting $d_{i}=\operatorname{dim} H_{i}$, we can define the space

$$
\mathfrak{E}_{1}(I)=\left\{\psi \in \prod_{i \in I} \mathscr{L}\left(H_{i}\right): \sum_{i \in I} d_{i}\|\psi(i)\|_{1}<\infty\right\} .
$$

Then with the pointwise operations of addition and multiplication, and the norm $\|\psi\|_{1}=\sum_{i \in I} d_{i}\|\psi(i)\|_{1}$, the space $\mathfrak{E}_{1}(I)$ is a Banach algebra. Moreover, we have $\mathfrak{E}_{1}(I)^{*} \cong \mathfrak{E}_{\infty}(I):=\ell^{\infty}-\bigoplus_{i \in I} \mathscr{L}\left(H_{i}\right)$ under the duality $\langle\psi, \varphi\rangle=\sum_{i \in I} d_{i} \operatorname{tr}(\psi(i) \varphi(i))$ (Hewitt and Ross [18, Theorem 28.31]).

Theorem 4.5. Let $\left\{\left(\pi_{i}, H_{i}\right)\right\}_{i \in I}$ be as above, $\alpha_{A}^{*}: U(A)^{*} \rightarrow A^{*}$ be the adjoint of $\alpha_{A}$, and $\psi \in \mathfrak{E}_{1}(I)$. Then

$$
\alpha_{A}^{*}(\psi)=\sum_{i \in I} d_{i} \operatorname{tr}\left(\psi(i) \pi_{i}\right) \in \operatorname{AP}(A)
$$

where the series is absolutely convergent.

Proof. First we prove that $\sum_{i \in I} d_{i} \operatorname{tr}\left(\psi(i) \pi_{i}\right) \in \operatorname{AP}(A)$. Since for every $i \in I, \pi_{i}$ is a continuous finite-dimensional representation of $A$, we have $\operatorname{tr}\left(\psi(i) \pi_{i}\right) \in \operatorname{AP}(A)$ [15, Lemma 2.3]. For every $b \in A$ we have

$$
\left|\left\langle\operatorname{tr}\left(\psi(i) \pi_{i}\right), b\right\rangle\right|=\left|\operatorname{tr}\left(\psi(i) \pi_{i}(b)\right)\right| \leq\left\|\pi_{i}(b)\right\|\|\psi(i)\|_{1} \leq\|b\|\|\psi(i)\|_{1}
$$

(for the first inequality, see [18, Theorem D.39(ii)]). By taking the supremum of the left hand side over all $b$ in the closed unit ball of $A$, it follows that $\left\|\operatorname{tr}\left(\psi(i) \pi_{i}\right)\right\|_{A^{*}} \leq\|\psi(i)\|_{1}$. Thus

$$
\sum_{i \in I} d_{i}\left\|\operatorname{tr}\left(\psi(i) \pi_{i}\right)\right\|_{A^{*}} \leq \sum_{i \in I} d_{i}\|\psi(i)\|_{1}=\|\psi\|_{1}<\infty,
$$


thus the series in 12 is absolutely convergent, and $\sum_{i \in I} d_{i} \operatorname{tr}\left(\psi(i) \pi_{i}\right) \in$ $\operatorname{AP}(A)$. It remains to prove the equality in $(12)$. Since $\psi \in \mathfrak{E}_{1}(I) \subset\left(\mathfrak{E}_{\infty}(I)\right)^{*}$, restricting $\psi$ to $U(A)$ defines an element in $U(A)^{*}$; we denote it by $\psi$ as well. Now for every $a \in A$,

$$
\begin{aligned}
\left\langle\alpha_{A}^{*}(\psi), a\right\rangle_{A^{*}, A} & =\left\langle\psi, \alpha_{A}(a)\right\rangle=\sum_{i \in I} d_{i} \operatorname{tr}\left(\psi(i) \alpha_{A}(a)(i)\right)=\sum_{i \in I} d_{i} \operatorname{tr}\left(\psi(i) \pi_{i}(a)\right) \\
& =\sum_{i \in I} d_{i}\left\langle\operatorname{tr}\left(\psi(i) \pi_{i}\right), a\right\rangle=\left\langle\sum_{i \in I} d_{i} \operatorname{tr}\left(\psi(i) \pi_{i}\right), a\right\rangle
\end{aligned}
$$

which proves the equality in 12 .

Let $\mathscr{A}_{0}(A)$ be the subalgebra of $A$ consisting of all $a \in A$ such that $\alpha_{A}(a) \in \mathfrak{E}_{1}(I)$, that is, $\mathscr{A}_{0}(A)$ is the set of all $a \in A$ such that $\left\|\alpha_{A}(a)\right\|_{1}=$ $\sum_{i \in I} d_{i}\left\|\pi_{i}(a)\right\|_{1}<\infty$. Let $\kappa: \mathfrak{E}_{1}(I) \rightarrow U(A)^{*}$ be the linear mapping that sends each element of $\mathfrak{E}_{1}(I)$ canonically into $\left(\mathfrak{E}_{\infty}(I)\right)^{*}$ and subsequently restricts it to $U(A)$. In view of Theorem 4.5, we can define a linear map

$$
\eta_{0}=\alpha_{A}^{*} \circ \kappa \circ \alpha_{A}: \mathscr{A}_{0}(A) \rightarrow \operatorname{AP}(A), \quad \eta_{0}(a)=\sum_{i \in I} d_{i} \operatorname{tr}\left(\pi_{i}(a) \pi_{i}\right),
$$

where the sum is absolutely convergent. Let $J=\left\{a \in \mathscr{A}_{0}(A): \alpha_{A}(a)=0\right\}$. Then $J$ is an ideal of $\mathscr{A}_{0}(A)$ and $J \subset$ ker $\eta_{0}$, therefore $\eta_{0}$ induces a linear map from the quotient algebra $\mathscr{A}_{0}(A) / J$ into $A^{*}$ which we denote by $\eta$ :

$$
\eta: \mathscr{A}_{0}(A) / J \rightarrow A^{*}, \quad a+J \mapsto \sum_{i \in I} d_{i} \operatorname{tr}\left(\pi_{i}(a) \pi_{i}\right) .
$$

If we equip the algebra $\mathscr{A}_{0}(A) / J$ with the norm

$$
\|a+J\|=\left\|\alpha_{A}(a)\right\|_{1}=\sum_{i \in I} d_{i}\left\|\pi_{i}(a)\right\|_{1},
$$

then by 13,

$$
\|\eta(a+J)\|_{A^{*}} \leq \sum_{i \in I} d_{i}\left\|\operatorname{tr}\left(\pi_{i}(a) \pi_{i}\right)\right\|_{A^{*}} \leq\left\|\alpha_{A}(a)\right\|_{1}=\|a+J\|,
$$

which shows that $\|\eta\| \leq 1$. Hence $\eta$ has a continuous linear extension (with the same norm) from the Banach algebra completion of $\mathscr{A}_{0}(A) / J$ into $A^{*}$. Let us denote the completion of $\mathscr{A}_{0}(A) / J$ by $\mathscr{A}(A)$, and the extended map by $\eta$ again. We can summarize the above arguments in the following theorem.

TheOREM 4.6. If $A$ is a Banach algebra, then there exists a linear mapping

$$
\eta: \mathscr{A}(A) \rightarrow \operatorname{AP}(A), \quad a+J \mapsto \sum_{i \in I} d_{i} \operatorname{tr}\left(\pi_{i}(a) \pi_{i}\right),
$$

with $\|\eta\| \leq 1$. 
We conclude by considering the important case of the group algebra $L^{1}(G)$, for which the map $\eta$ in 15$)$ can be related to the Fourier series. For details of harmonic analysis on compact groups we refer the readers to [18, §34].

EXAMPLE 4.7. Let $G$ be a compact group and $L^{1}(G)$ be the group algebra of $G$ under the convolution product. As we saw in Example 3.2 , the set of all nonzero finite-dimensional irreducible involutive representations of $L^{1}(G)$ can be written as $\left\{\left(\bar{\pi}^{\prime}, H_{\pi}\right)\right\}_{\pi \in \widehat{G}}$. Furthermore, by (8), if $f \in L^{1}(G)$ and $\pi \in \widehat{G}$, then $\alpha_{L^{1}}(f)(\pi)=\widehat{f}(\pi)$, and thus $\mathscr{A}_{0}\left(L^{1}(G)\right)$ is the set of all functions $f \in L^{1}(G)$ such that $\left\|\alpha_{L^{1}}(f)\right\|_{1}=\sum_{\pi \in \widehat{G}} d_{\pi}\|\widehat{f}(\pi)\|_{1}<\infty$. It follows from the injectivity of the Fourier transform that the RFD transform $\alpha_{L^{1}}$ is injective. In addition, it is known that the space $\mathscr{A}_{0}\left(L^{1}(G)\right)$ is complete under the norm $\|f\|=\left\|\alpha_{L^{1}}(f)\right\|_{1}$ [18, Corollary 34.7], and hence $\mathscr{A}\left(L^{1}(G)\right)=\mathscr{A}_{0}\left(L^{1}(G)\right)$. An important result in harmonic analysis states that every $f \in \mathscr{A}\left(L^{1}(G)\right)$ is equal (almost everywhere) to its Fourier series, i.e., to the continuous function $S(f)=\sum_{\pi \in \widehat{G}} d_{\pi} \operatorname{tr}\left(\widehat{f}(\pi)^{t} \pi\right)$ [18, Corollary 34.6], where by (13) we have $\sum_{\pi \in \widehat{G}} d_{\pi}\left\|\operatorname{tr}\left(\widehat{f}(\pi)^{t} \pi\right)\right\|_{\infty}<\infty$. Thus $\mathscr{A}\left(L^{1}(G)\right)$ is the Banach algebra (under convolution) of functions in $L^{1}(G)$ with absolutely convergent Fourier series. The mapping $\eta$ defined in 15 can be characterized as follows. For $f \in L^{1}(G)$, let $\check{f} \in L^{1}(G)$ be defined as $\check{f}(x)=f\left(x^{-1}\right)(x \in G)$. Since a representation $\pi \in \widehat{G}$ and its associated representation $\pi^{\prime}$ have the same coordinate functions, it follows that

$$
\operatorname{tr}\left(\pi^{\prime}(\check{f}) \pi^{\prime}\right)=\operatorname{tr}\left(\pi^{\prime}\left(\overline{f^{*}}\right) \pi^{\prime}\right)=\operatorname{tr}\left(\pi^{\prime}(\bar{f})^{*} \pi^{\prime}\right)=\operatorname{tr}\left(\bar{\pi}^{\prime}(f)^{t} \pi\right)=\operatorname{tr}\left(\widehat{f}(\pi)^{t} \pi\right) .
$$

Therefore

$$
\eta(\check{f})=\sum_{\pi \in \widehat{G}} d_{\pi} \operatorname{tr}\left(\pi^{\prime}(\check{f}) \pi^{\prime}\right)=\sum_{\pi \in \widehat{G}} d_{\pi} \operatorname{tr}\left(\widehat{f}(\pi)^{t} \pi\right)=S(f) .
$$

Thus $\eta(\check{f})$ is the Fourier series of $f$ and $\eta(\check{f})=f$ for all $f \in \mathscr{A}\left(L^{1}(G)\right)$.

REMARK 4.8. In recent years, a great interest in the space $\mathscr{A}\left(L^{1}(G)\right)$ has arisen from the fact that upon identifying each $f \in \mathscr{A}\left(L^{1}(G)\right)$ with its Fourier series $S(f)$, the space $\mathscr{A}\left(L^{1}(G)\right)$ forms a Banach algebra of continuous functions under pointwise operations of addition and multiplication, and the norm $\|f\|=\left\|\alpha_{L^{1}}(f)\right\|_{1}$ (a fact first proved by Krein [20] for $G$ compact). This Banach algebra is usually denoted by $A(G)$ and called the Fourier algebra of $G$. The analogue of this algebra for general locally compact groups was introduced by Eymard [13, and has been studied extensively by many authors. For a survey on Fourier algebras we refer the readers to a forthcoming book by Kaniuth and Lau [19]. 
Acknowledgements. The second author was supported by an NSERC grant.

The preparation of the paper started when the first author was visiting University of Windsor in April 2013 and February 2015. He would like to thank Mehdi Monfared for his warm hospitality, and the Department of Mathematics in Windsor. The authors thank F. Ghahramani, B. Forrest, N. Spronk, M. Wiersma and Z. Tanko for helpful discussions.

\section{References}

[1] R. J. Archbold, On residually finite-dimensional $C^{*}$-algebras, Proc. Amer. Math. Soc. 123 (1995), 2935-2937.

[2] B. Blackadar, Operator Algebras. Theory of $C^{*}$-algebras and von Neumann algebras, Springer, New York, 2006.

[3] M. D. Choi, The full $C^{*}$-algebra of the free group on two generators, Pacific J. Math. 87 (1980), 41-48.

[4] M. Dadarlat, Residually finite-dimensional $C^{*}$-algebras, in: Operator Algebras and Operator Theory (Shanghai, 1997), Contemp. Math. 228, Amer. Math. Soc., Providence, RI, 1998, 45-50.

[5] H. G. Dales, Banach Algebras and Automatic Continuity, Oxford Univ. Press, Oxford, 2000.

[6] H. G. Dales and A. T.-M. Lau, The second duals of Beurling algebras, Mem. Amer. Math. Soc. 177 (2005), no. 836.

[7] J. Dixmier, Les $C^{*}$-Algèbres et Leurs Représentations, 2ème éd., Éditions Jacques Gabay, Paris, 1996.

[8] J. Duncan and S. A. R. Hosseiniun, The second dual of a Banach algebra, Proc. Roy. Soc. Edinburgh Sect. A 84 (1979), 309-325.

[9] J. Duncan and A. Ülger, Almost periodic functionals on Banach algebras, Rocky Mountain J. Math. 22 (1992), 837-848.

[10] N. Dunford and J. T. Schwartz, Linear Operators, Part I: General Theory, Wiley, New York, 1988.

[11] C. F. Dunkl and D. E. Ramirez, Subalgebras of the dual of the Fourier algebra of a compact group, Proc. Cambridge Philos. Soc. 71 (1972), 329-333.

[12] R. Exel and T. A. Loring, Finite-dimensional representations of free product $C^{*}$ algebras, Int. J. Math. 3 (1992), 469-476.

[13] P. Eymard, L'algèbre de Fourier d'un groupe localement compact, Bull. Soc. Math. France 92 (1964), 181-236.

[14] M. Filali, M. Neufang and M. Sangani Monfared, Representations of Banach algebras subordinate to topologically introverted spaces, Trans. Amer. Math. Soc. 367 (2015), 8033-8050.

[15] M. Filali and M. Sangani Monfared, Finite-dimensional left ideals in the duals of introverted spaces, Proc. Amer. Math. Soc. 139 (2011), 3645-3656.

[16] K. R. Goodearl and P. Menal, Free and residually finite-dimensional $C^{*}$-algebras, J. Funct. Anal. 90 (1990), 391-410.

[17] E. E. Granirer, On some spaces of linear functionals on the algebras $A_{p}(G)$ for locally compact groups, Colloq. Math. 52 (1987), 119-132.

[18] E. Hewitt and K. A. Ross, Abstract Harmonic Analysis, Vol. II, Springer, Berlin, 1970. 
[19] E. Kaniuth and A. T.-M. Lau, Fourier and Fourier-Stieltjes Algebras on Locally Compact Groups, to appear.

[20] M. G. Krein, Hermitian-positive kernels on homogeneous spaces, I, II, Ukrain. Mat. Zh. 1 (1949), no. 4, 64-98, and 2 (1950), no. 1, 10-59 (in Russian); English transl.: Amer. Math. Soc. Transl. Ser. II, 34 (1963), 69-108, 109-164.

[21] A. T.-M. Lau and J. C. S. Wong, Weakly almost periodic elements in $L_{\infty}(G)$ of a locally compact group, Proc. Amer. Math. Soc. 107 (1989), 1031-1036.

[22] H. Lin, Residually finite-dimensional and AF-embeddable $C^{*}$-algebras, Proc. Amer. Math. Soc. 129 (2001), 1689-1696.

[23] T. W. Palmer, Banach Algebras and the General Theory of *-Algebras, Vol. 1, Cambridge Univ. Press, Cambridge, 1994.

[24] V. G. Pestov, Operator spaces and residually finite-dimensional $C^{*}$-algebras, J. Funct. Anal. 123 (1994), 308-317.

[25] M. Takesaki, Theory of Operator Algebras I, Springer, New York, 2001.

[26] N. J. Young, Periodicity of functionals and representations of normed algebras on reflexive spaces, Proc. Edinburgh Math. Soc. 20 (1976), 99-120.

M. Filali

Department of Mathematical Sciences

University of Oulu

Oulu 90014, Finland

E-mail: mahmoud.filali@oulu.fi
M. Sangani Monfared

Department of Mathematics and Statistics

University of Windsor

Windsor, ON, N9B 3P4, Canada

E-mail: monfared@uwindsor.ca 